\title{
A fractal model of normal dynamic parameters for fixed oily porous media joint interface in machine tools
}

\author{
Chongnian Qu $\cdot$ Liangsheng $\mathrm{Wu} \cdot$ Jianfeng Ma • \\ Qun Xia • Shuhui Ma
}

Received: 29 October 2012 / Accepted: 29 January 2013 /Published online: 21 February 2013

(C) The Author(s) 2013. This article is published with open access at Springerlink.com

\begin{abstract}
The joint interface plays a significant role in machine tools and other machineries. A novel type of joint which consists of oily porous material was proposed. The equivalent stiffness and damping of oily porous joint could be divided into solid and liquid parts, and the two parts are shunt connection. Theoretically, the stiffness and damping of solid part were established using Hertz contact and fractal theory, and the liquid counterpart was also deduced from the average flow of generalized Reynolds equation through a correlation parameter $A_{\mathrm{r}}$. It was concluded from simulation and calculation that the total equivalent stiffness of oily joint is increased with $D$ and decreased with increased $G$. The experiment was carried out to compare the normal characteristics of porous material-steel oily joint with non-media steel-steel joint, and the result was that the joint which contains an oil film interlayer formed by porous and steel is superior to the joint with non-media formed by steel in stiffness and damping characteristics. In the case of the same preload, the former's stiffness is increased by about $30 \%$, and the damping is increased about five to six times meanwhile.
\end{abstract}

Keywords Fractal theory $\cdot$ Stiffness $\cdot$ Damping $\cdot$ Joint interface $\cdot$ Machine tools

\section{Introduction}

A whole machine tool structure, unlike a single structure or a component, is composed of many components connected with many joint interfaces. The various types of joint interfaces

C. Qu $(\bowtie) \cdot \mathrm{L} . \mathrm{Wu} \cdot \mathrm{J} . \mathrm{Ma} \cdot \mathrm{Q} . \mathrm{Xia} \cdot \mathrm{S}$. Ma

College of Mechanical Engineering and Applied Electronics

Technology, Beijing University of Technology,

Beijing 100124, China

e-mail: qchnian@126.com play very significant roles in an integrated machine tool structure. Obviously, the transmission of force and precision between machine tool structures are related to the status and performance of joint interfaces. Therefore, the research of the joint interface is the foundation study of the mechanical properties in machine tool [1-3]. In reference [4], Bartosz Powałka and Tomasz Okulik presented a new technology for the assembly of ball guideway systems which involve the use of a thin layer of a casting compound to suppress vibration and chatter in machine tools. Since $40-60 \%$ of the total dynamic stiffness and about $90 \%$ of the total damping in a whole machine tool structure originate in joint interfaces [5-8], it is beneficial to design and build high stiffness and damping joint interface for the mechanical properties of an integrated machine tool to improve the cutting machining accuracy.

As well known, the contact state of the joint can be well improved by taking lubricating oil or grease as addition in the joint interface, and the stiffness and damping are increased meanwhile [9-11]. Oil-soaked porous material can be used as one or both parts of the joint interface and even as the middle of the sandwich structure (as shown in Fig. 1).

In a working condition, the lubricating oil in the porous material exudes and fills in the joint interface due to external excitation and elevated temperature. While stopping, a part of the oil in the joint interfaces may seep back into the porous material when the loads disappear.

However, the dynamic characteristic of the oily joint interface mentioned above is complex due to hybrid contact of liquid and solid; it is difficult to quantitatively demonstrate the dynamic parameters of the joint interface. The development of fractal theory makes it feasible to explain the mechanism of this kind of joint interface consisting of porous material $[12,13]$.

In this paper, a novel type of joint which consisted of oily porous material was proposed. The normal modal based on fractal contact theory was established, which divided the 
oil-soaked porous Joint surface
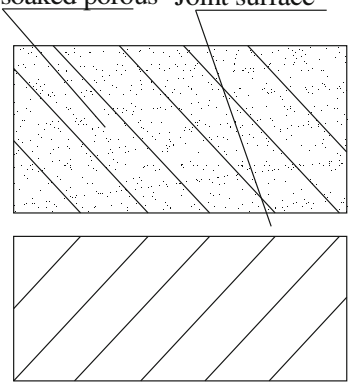

(a) oil-soaked porous Joint surface

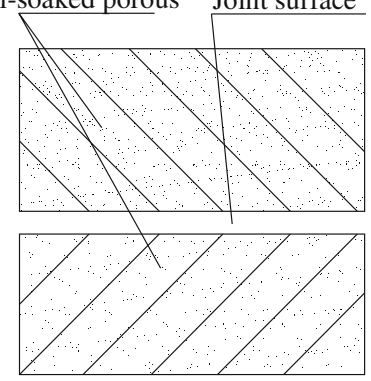

(b)

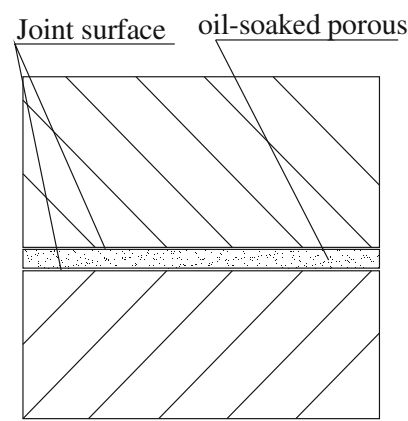

(c)

Fig. 1 Joint interfaces consist of oil-soaked porous material: a porous as one part; $\mathbf{b}$ porous as both parts; $\mathbf{c}$ porous as middle in sandwich structure

stiffness of oily fixed joint interface into the solid contact stiffness and the liquid film dynamic stiffness, and the two parts were shunt connection. Thereby the comprehensive stiffness modal for the joint interface which consisted of porous material was derived. The damping coefficient modal of the joint interface was developed in accordance with the same method. The advantage of this kind of joint is not only increasing the stiffness and damping, but also filling oil for a considerable long period is not needed. High stiffness and damping are essential for machine tools because the higher stiffness may directly increase the first-order natural frequency, which may improve the precision of the machined surface. Furthermore, the higher stiffness and damping may play an important role for reducing the receptance between the tool tip and the workpiece to minimize vibration and chatter.

\section{Shunt contact modal}

At the micro level, the joint interface could be considered as a mixture which contains some convex peaks and concave valleys whose size is randomly distributed due to the machining method restrictions (as shown in Fig. 2).

When contact happens, it must be that the higher convex peaks meet each other at first between two

surfaces. The protruding peaks become flexibility (even plastic) deformation under the loadings. Meanwhile, oil soaked in the porous material exudes partly due to the changes of the pores volume and fills in the concave valley at the two contact surfaces, and the oil leaks out at the contact boundary, as shown in Fig. 1b, c. The normal load is supported by both solid and liquid contact areas. Then, the characteristics of stiffness and damping of the joint interface depend on the counterpart of the asperity and the micro-pressure oil film in the constrained space.

In most cases, the contact of the joint interface contains two parts: solid one and liquid one. Normal load is supported by both of them. Usually, joint interface can be equivalent to a stand-alone dynamic unit, which contains the mass, the stiffness, the damping coefficient, and the distribution rule of these parameters. Because the convex peak of the surface is tiny, the mass of the joint interface unit can be neglected, and then the dynamic parameters of the joint unit can be considered to contain only the stiffness and the damping. Thus, the stiffness of the joint can be divided into the solid stiffness and the liquid dynamic stiffness, and the two parts are shunt connection [14], and so does the damping coefficient. It is assumed that the oil in the porous material was seeped and filled in the concave valleys of the surface meanwhile. The hypothesis is consistent with the reality mostly $[15,16]$.

Generally, finite element was used to calculate the mechanical properties of the joint interface, where the general simplified method is to simplify as one or several integrated points, not to consider the mutual influence of the joint and its base. This simplifying method can be used only in the situation if the stiffness of a joint base is greatly bigger than the stiffness of the joint interface; in other words, in the situation when the vibrating mass is horizontally movable. Thus, we could select an appropriate contact area to consider that the vibrating mass is horizontally movable for theoretical analysis, which would also make it easy to carry out the experiment, and then the joint interface could be described as Fig. 3.

In Fig. 3, $K_{\mathrm{s}}$ is the total stiffness of solid part, $K_{\mathrm{L}}$ is the total stiffness of liquid part, $C_{\mathrm{s}}$ is the total damping coefficient of solid part, and $C_{\mathrm{L}}$ is the total damping coefficient of liquid part. Considering shunt connection, the comprehensive equivalent stiffness and damping of the joint interface can be described as Eq. (1).

$\left\{\begin{array}{l}K=K_{S}+K_{L} \\ C=C_{S}+C_{L}\end{array}\right.$

In Eq. (1), $K$ and $C$ are the comprehensive equivalent stiffness and damping of the joint interface consists of porous material. 
Fig. 2 Schematic diagram of contact with oily porous surface: a before contact; b after contact; c contact partially enlarged

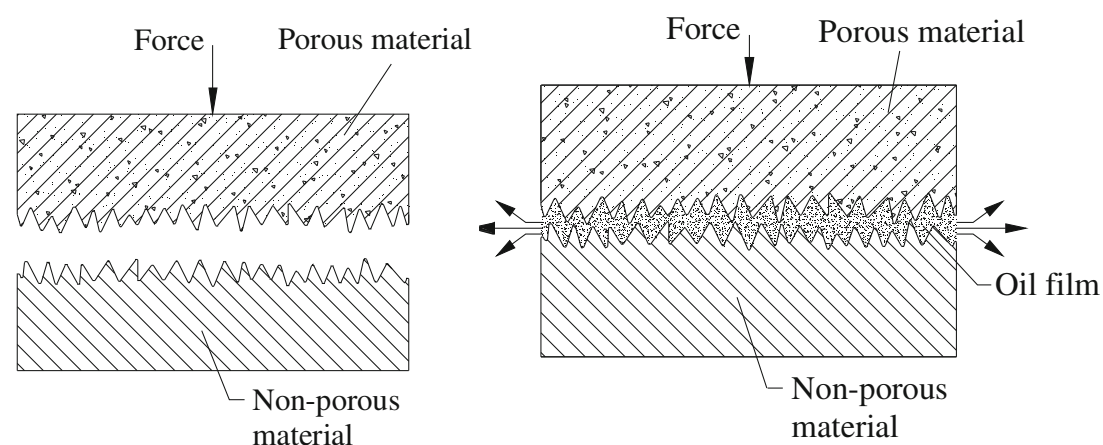

(a)

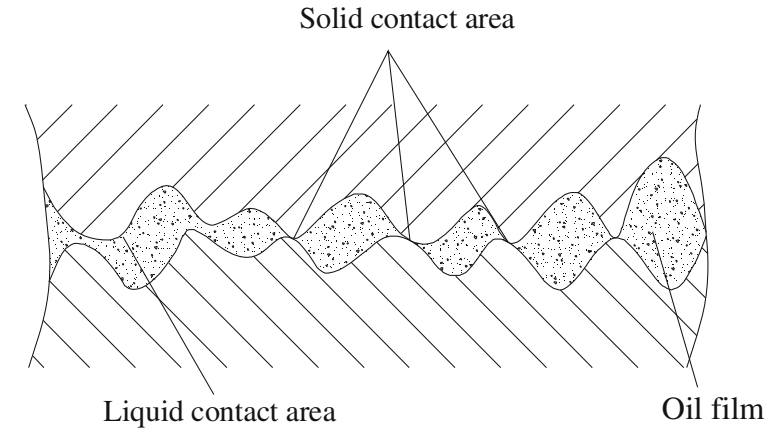

(c)

\section{Fractal modal of stiffness}

\subsection{Normal stiffness of solid}

According to Hertz theory and Majumdar-Bhushan (M-B) fractal contact modal $[17,18]$, the critical area of a single convex peak while it yields elastic-plastic deformation complies with Eq. (2):

$a_{c}=G^{2}(2 E / H)^{2 /(D-1)}$

where $D(1<D<2)$ is the fractal dimension of the surface contour, $G$ is the fractal roughness coefficient, $E$ is the comprehensive elastic modulus of the joint interface, and $H$ is the hardness of the softer material. Wherein, $E$ can be defined by the formula

$f(t)$

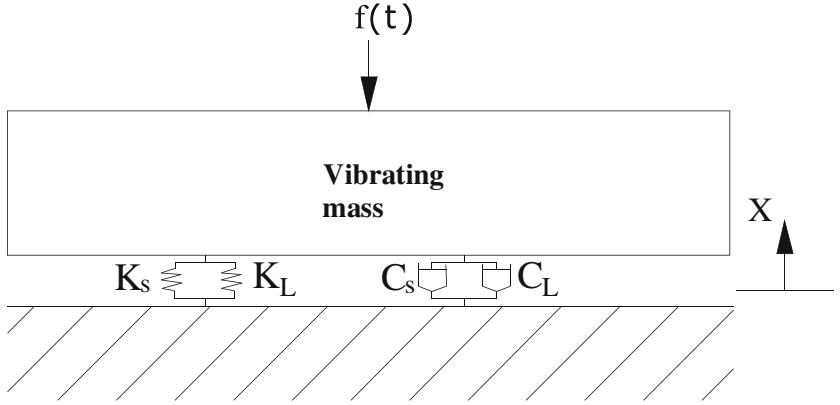

Fig. 3 Shunt modal of joint interface
$E^{-1}=\left(1-v_{1}^{2}\right) E_{1}^{2}+\left(1-v_{2}^{2}\right) E_{2}^{2}, E_{1}, E_{2}, v_{1}, v_{2}$

which is the elastic modulus and Poisson's ratio of two parts which consist the joint interface, respectively.

According to the Hertz theory, when the contact area of a single point is greater than the critical area $a_{\mathrm{c}}$, the contact point is elastically deformed and shows stiffness characteristics, while the contact area of a single point is smaller than the critical area $a_{\mathrm{c}}$, the plastic deformation occurs, and the contact point shows damping characteristics. For a point of contact area $a$, as shown in Fig. 4, the radius of the tiny convex peak is $R$, and the radius of the contact area is $r$. Under the normal load $P(a)$, the normal contact deformation is $\delta$. Based on Hertz theory and fractal contact theory, all the parameters satisfy the following formulas [19].

$P(a)=\frac{4}{3} E R^{\frac{1}{2}} \delta^{\frac{3}{2}}$

$R=\frac{a^{D / 2}}{\pi^{2} G^{D-1}}$

$r=\left(\frac{3 P(a) R}{4 E}\right)^{\frac{1}{3}}$

$a=\pi r^{2}=\pi R \delta$ 
When $a$ is greater than $a_{\mathrm{c}}$, elastic deformation occurs, and then, the normal contact load of one convex peak is

$P_{e}(a)=\frac{4 \sqrt{\pi}}{3} E G^{D-1} a^{3-D / 2}$

When $a$ is smaller than $a_{\mathrm{c}}$, plastic deformation occurs, and then the normal contact load of one convex peak is

$P_{p}(a)=H a$

From the Eqs. (3)-(6), the normal stiffness of one convex peak can be derived, which is,

$k_{n}=\frac{d P_{a}}{d \delta}=2 E R^{\frac{1}{2}} \delta^{\frac{1}{2}}=2 E \sqrt{\frac{a}{\pi}}$

And then the comprehensive stiffness of the solid contact, that is, the stiffness from all the convex peaks is,

$K_{s}=\int_{a_{c}}^{a_{L}} k_{n} n(a) d a$

Meanwhile the normal load, which is contains elastic and plastic load is,

$P=\int_{a_{c}}^{a_{L}} n(a) P_{e}(a) d a+\int_{0}^{a_{c}} n(a) P_{p}(a) d a$

wherein, $a_{\mathrm{L}}$ is the biggest convex peak on the contact surface, and $n(a)$ is the distribution function of all the peaks on the contact surface. In reference [13], $n(a)$ is a power-law function, and it can be quoted from the M-B modal, as shown in Eq. (12)

$n(a)=\frac{D}{2} \frac{a_{L}^{D / 2}}{a^{D / 2+1}}$

Substitute Eqs. (9) and (12) into Eq. (10), the comprehensive stiffness of solid contact is derived, which is

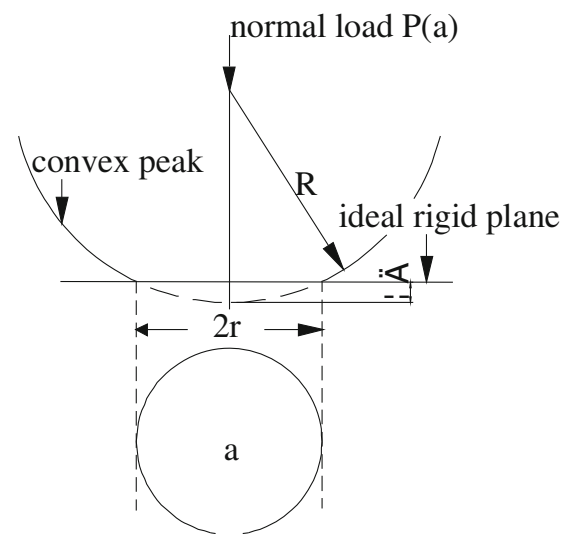

Fig. 4 Simplified schematic of convex peak contact
$K_{s}=\frac{2 D E}{\sqrt{\pi}(1-D)}\left(a_{L}^{1 / 2}-a_{L}^{D / 2} a_{c}^{(1-D) / 2}\right)$

From Eq. (12), we can get the real contact area $A_{\mathrm{r}}$,

$A_{r}=\int_{0}^{a_{L}} n(a) d a=\frac{D}{2-D} a_{L}$

That is, $a_{\mathrm{L}}=(2-D) A_{\mathrm{r}} / D$

Substitute $a_{\mathrm{c}}, a_{\mathrm{L}}$ (Eqs. 2 and 14) into the Eq. (13), the modal of comprehensive stiffness of solid contact of the porous joint interface can be obtained as,

$K_{s}=f_{1}(D) A_{r}^{\frac{1}{2}}\left(1-f_{2}(D) A_{r}^{\frac{D-1}{2}}\right)$

Wherein,

$f_{1}(D)=\frac{2 E D}{(1-D)} \sqrt{\frac{2-D}{\pi D}}, f_{2}(D)=\frac{2 E G^{D-1}}{H}\left(\frac{2-D}{D}\right)^{\frac{D-1}{2}}$

Through Eqs. (11) and (12), the total elastic-plastic load can be evaluated as

$\begin{cases}P=\frac{4 \sqrt{\pi}}{3} E G^{D-1} g_{1}(D) A_{r}^{\frac{D}{2}}\left[\left(\frac{2-D}{D}\right)^{\frac{3-2 D}{2}}-a_{c}^{\frac{3-2 D}{2}}\right]+H g_{2}(D) A_{r}^{\frac{D}{2}} a_{c}^{\frac{2-D}{2}}(D \neq 1.5) \\ P=E G^{\frac{1}{2}} A_{r}^{\frac{3}{4}} \ln \left(\frac{A_{c}}{a_{c}}\right)+H A_{r}^{\frac{3}{4}} a_{c}^{\frac{1}{4}} & (D=1.5)\end{cases}$

where,

$g_{1}(D)=\frac{D}{3-2 D}\left(\frac{2-D}{D}\right)^{\frac{D}{2}}, g_{2}(D)=\left(\frac{D}{2-D}\right)^{\frac{2-D}{2}}$.

Equation (15) shows the nonlinear relationship between the normal stiffness of solid and the real solid contact area. Since the real solid contact area is associated to the normal load (see Eq. 16), the Eqs. (15) and (16) demonstrate the implied relationship between the normal stiffness and load.

\subsection{Normal damping of solid}

As mentioned above, while the contact area of a single point is smaller than the critical area $a_{\mathrm{c}}$, the plastic deformation occurs, and the contact point shows damping characteristics. And then the plastic strain energy can be evaluated as,

$w_{p}=\frac{H D G^{D-1}}{4(2-D)} a_{L}^{D / 2} a_{c}^{2-D}$

Similarly, the elastic strain energy generated in the elastic contact area can be evaluated as

$w_{e}=\frac{8 E D G^{2(D-1)}}{15 \sqrt{\pi}(5-3 D)}\left(a_{L}^{(5-2 D) / 2}-a_{L}^{D / 2} a_{c}^{(5-3 D) / 2}\right)$ 


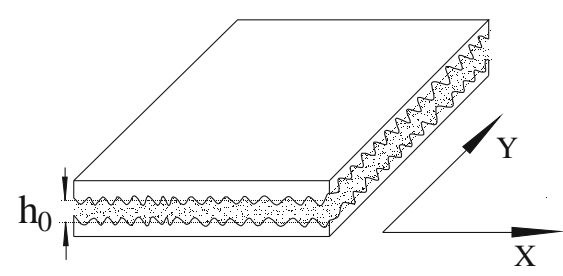

Fig. 5 Oily joint interface

Therefore, the damping loss factor can be deduced through Eqs. (17) and (18), which is,

$\xi=w_{p} / w_{e}$

Assuming that the mass of the substrate (which contains the rough surface) is $m$, then the normal damping coefficient is,

$C_{s}=\xi\left(m K_{n}\right)^{\frac{1}{2}}$

Equation (20) illustrates that the normal damping coefficient is determined by normal load, material properties of the rough surface, and fractal parameters. Equations (17)-(20) constitute the normal damping fractal modal of the joint interface.

\subsection{Equivalent stiffness of liquid}

In the working condition, the oil in the porous material is exuded because of external excitation and filled in the concave valleys between the joint interfaces. The contact area is divided into two solid and liquid parts. Thus, the liquid contact area is $A_{\mathrm{o}}=A-A_{\mathrm{r}}$, where, $A_{\mathrm{o}}$ means oil contact area.

According to the average flow of generalized Reynolds equation, not considering lateral leakage, the average
Reynolds equation only contains extruded items and is shown as Eq. (21),

$\frac{\partial}{\partial x}\left(\varphi_{x} \frac{\partial p}{\partial x}\right)+\frac{\partial}{\partial y}\left(\varphi_{y} \frac{\partial p}{\partial y}\right)=-\varphi_{c} \frac{12 \eta}{h^{3}} \frac{\partial h}{\partial t}$

In Eq. (21), the parameters $\varphi_{x}$ and $\varphi_{y}$ are pressure flow factors at directions $x$ and $y$, respectively, $\varphi_{c}$ is contact factor, $\eta$ is fluid dynamic viscosity, and $h$ is the oil film thickness; its initial value is assumed as the distance between the rough midline under a certain normal load, that is, nominally the thickness of the oil film h0 (shown in Fig. 5). Assuming the contact surface of the joint interface is isotropic, then $\varphi_{x}=\varphi_{y}$

The parameter $\varphi_{x}$ represents the ratio of the average lubricating oil flow rate between rough surface and the corresponding smooth surface. Considering the relationship between real contact area $A_{\mathrm{r}}$, the nominal contact area $A$, and the lubricating oil flow rate, the parameter $\varphi_{x}$ is defined as $\varphi_{x}=A_{\mathrm{r}} / A$.

The parameter $\varphi_{c}$ represents the proportion of noncontact in the rough surface. In oily joint interface, that is, $\varphi_{c}$ is the proportion of liquid contact throughout the nominal contact area. It can be defined as $\varphi_{c}=A_{\mathrm{o}} / A=\left(A-A_{\mathrm{r}}\right) / A$.

The average Reynolds equation only contains $x$-direction is

$\frac{d^{2} p}{d x^{2}}=-\frac{A-A_{r}}{A_{r}} \frac{12 \eta}{h^{3}} \frac{\partial h}{\partial t}$

By solving Eq. (22) and simplifying the liquid contact area in joint interface as circular contact, the load carrying capacity of oil film, that is, the anti-squeeze force of oil can be obtained as

$W=-\frac{A-A_{r}}{A_{r}} \frac{3 \pi \eta}{2 h^{3}}\left(\frac{d h}{d t}\right) R^{\prime} 4$
Table 1 Solid and liquid contact dynamic parameter modal

\begin{tabular}{|c|c|}
\hline Solid stiffness & $K_{s}=f_{1}(D) A_{r}^{\frac{1}{2}}\left(1-f_{2}(D) A_{r}^{\frac{D-1}{2}}\right)$ \\
\hline Solid damping & $C_{s}=\xi\left(m K_{n}\right)^{\frac{1}{2}}$ \\
\hline Liquid stiffness & $K_{L}=\frac{9}{2} \frac{\left(A-A_{r}\right)^{3} \beta_{1} \beta_{2} \eta v}{A_{r} \pi h^{4}}$ \\
\hline Liquid damping & $C_{L}=\frac{3}{2} \frac{\left(A-A_{r}\right)^{3}}{A_{r}} \frac{\eta \beta_{1} \beta_{2}}{\pi h^{3}}$ \\
\hline Total stiffness & $K=K_{s}+K_{L}=f_{1}(D) A_{r}^{\frac{1}{2}}\left(1-f_{2}(D) A_{r}^{\frac{D-1}{2}}\right)+\frac{9}{2} \frac{\left(A-A_{r}\right)^{3} \beta_{1} \beta_{2} \eta v}{A_{r} \pi h^{4}}$ \\
\hline Total damping & $C=C_{s}+C_{L}=\xi\left(m K_{n}\right)^{\frac{1}{2}}+\frac{3}{2} \frac{\left(A-A_{r}\right)^{3}}{A_{r}} \frac{\eta \beta_{1} \beta_{2}}{\pi h^{3}}$ \\
\hline Total load & $\begin{cases}P=\frac{4 \sqrt{\pi}}{3} E G^{D-1} g_{1}(D) A_{r}^{\frac{D}{2}}\left[\left(\frac{2-D}{D}\right)^{\frac{3-2 D}{2}}-a_{c}^{\frac{3-2 D}{2}}\right]+H g_{2}(D) A_{r}^{\frac{D}{2}} a_{c}^{\frac{2-D}{2}}(D \neq 1.5) \\
P=E G^{\frac{1}{2}} A_{r}^{\frac{3}{4}} \ln \left(\frac{A_{r}}{a_{c}}\right)+H A_{r}^{\frac{3}{4}} a_{c}^{\frac{1}{4}} & (D=1.5)\end{cases}$ \\
\hline
\end{tabular}




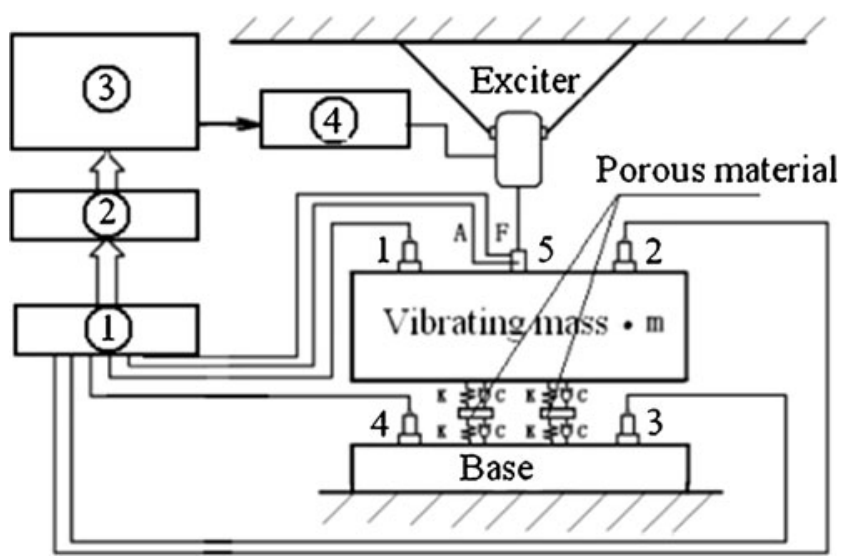

Fig. 6 Designed joint interface and experimental setup. (1, 2, 3, 4 are acceleration sensors, 5 is impedance head, (1) is charge amplifier, (2) is data acquisition card, (3) is computer, (4) is power amplifier, $A$ acceleration signal, $F$ force signal)

where, $R^{\prime}=\sqrt{\frac{A-A_{r}}{\pi}}, \frac{d h}{d t}$ is the normal vibrating speed of the joint interface, that is, $v=\frac{d h}{d t}$. Then Eq. (23) can be transformed into Eq. (24).

$W=-\frac{\left(A-A_{r}\right)^{3}}{A_{r}} \frac{3 \eta}{2 \pi h^{3}} v$

The derivation of Eq. (24) by the parameter $h$ is,

$K_{L}=\frac{d W}{d h}=\frac{9}{2} \frac{\left(A-A_{r}\right)^{3} \beta_{1} \beta_{2} \eta v}{A_{r} \pi h^{4}}$

Equation (25) shows the normal equivalent stiffness of oil contact, the parameter $\beta_{1}$ is shape factor [20], and $\beta_{1}=0.421$, while circular contact. $\beta_{2}$ is the factor introduced to modify the deviation caused by the jointed surface profile and topography, and it can be fitted from experiment results. In this paper, $\beta_{2}=0.7$. Equation (25) shows the relationship between the normal liquid dynamic stiffness, the vibrating velocity, the real contact area, the liquid viscosity, and the nominal oil film thickness. It also describes the relationship between the liquid dynamic stiffness and the normal load through the parameter $A_{\mathrm{r}}$. From Eq. (25), it can be concluded that the stiffness of liquid contact is dynamic under the role of normal vibrating velocity $v$. Meanwhile, the liquid stiffness is inversely proportional to the fourth power of $h$; the smaller film thickness, the greater the film dynamic stiffness contributes.

\subsection{Equivalent damping of liquid}

Similarly, the derivation of Eq. (24) by the parameter $v$ is,

$C_{L}=\frac{d W}{d v}=\frac{3}{2} \frac{\left(A-A_{r}\right)^{3}}{A_{r}} \frac{\eta \beta_{1} \beta_{2}}{\pi h^{3}}$

Equation (26) shows the relationship between the equivalent damping of liquid and real contact area, liquid viscosity, and nominal oil film thickness.

\subsection{Comprehensive fractal modal of joint interface}

From the above analysis and formula (1), the normal dynamic parameters in oily porous joint interface contain two parts, and the two parts are shunt connection. The comprehensive normal parameters can be summarized in Table 1 .

\section{Experimental setup and discussion}

The designed joint interface and experimental setup is shown in Fig. 6. The porous material is cut to be four little square shims, whose size is $10 \times 10 \times \mathrm{mm}$. They are centrosymmetric placed around the exciting point between the base and the vibrating mass.

According to the designed setup, the nominal contact area is $A=4 \times 10^{-4} \mathrm{~m}^{2}$. The values of other parameters are given as Table 2. Additionally, joint interface is pre-stressed in normal direction with two $M 10$ screws, and the initial pretightening torque is $10 \mathrm{Nm}$.

\subsection{Variation of the contact area}

While contact occurs, the real contact area is not equal to the nominal contact area. The main factors affecting the real contact area are fractal parameters and normal load. The variation of real contact area $A_{\mathrm{r}}$ along with fractal dimensions $D$ and $G$ is shown in Fig. 7. The real contact area $A_{\mathrm{r}}$ is merely a small part of the nominal contact area $A$, and $A_{\mathrm{r}}$ is reduced while $\mathrm{G}$ was increasing, that is, the coarser the surface is, the smaller the $A_{\mathrm{r}}$ is. Meanwhile, the real contact area $A_{\mathrm{r}}$ is increased along with increased $D$ at first and then decreased.

\subsection{Variation of equivalent stiffness}

As mentioned above, the equivalent stiffness of oily porous fixed joint interface consists of solid and liquid part. Variation
Table 2 Values of some parameters

\begin{tabular}{llllllll}
\hline Parameters & $H(\mathrm{~Pa})$ & $E(\mathrm{~Pa})$ & $G(\mathrm{~m})$ & $h(\mathrm{~m})$ & $\eta(\mathrm{Pa} \cdot \mathrm{s})$ & $v(\mathrm{~m} / \mathrm{s})$ & $m(\mathrm{~kg})$ \\
\hline Value & $9 \times 10^{9}$ & $2 \times 10^{11}$ & $10^{-11}-10^{-13}$ & $1 \times 10^{-5}$ & 0.018 & $3 \times 10^{-3}$ & 15 \\
\hline
\end{tabular}




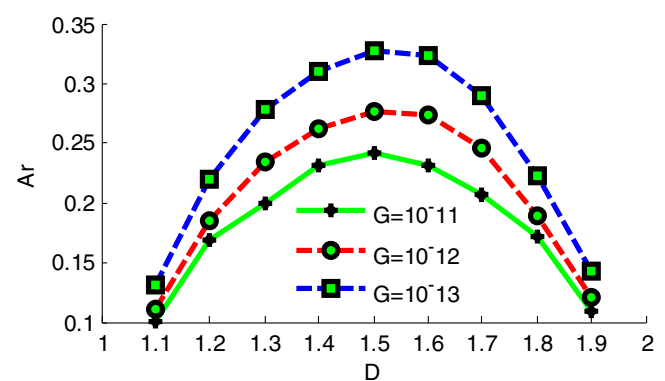

Fig. 7 Relationship of parameters $A_{\mathrm{r}}, D$, and $G$

of solid stiffness along with parameters $A_{\mathrm{r}}$ (percentage), $G$ (constant), and $D$ (variable) is shown in Fig. 8.

From Fig. 8a, the stiffness of solid part was increased with the real contact area $A_{\mathrm{r}}$ and the fractal dimension $D$ increased while the fractal roughness coefficient $G$ is a constant. On the contrary, it was increased when fractal roughness coefficient $G$ decreased while $D$ is a constant. $G$ decreased implies the surface roughness decreased. Thus, decreasing the surface roughness is beneficial to improve the stiffness characteristic of joint interface.

Variation of liquid stiffness along with parameters $A_{\mathrm{r}}$ (percentage), $G$ (constant), and $D$ (variable) is shown in Fig. 9. It is concluded from Fig. 9 that stiffness of liquid part has the same change trend when parameter $G$ is constant and $D$ is variable or under the opposite situation. That is, the stiffness of liquid part was decreased with the real contact area $A_{\mathrm{r}}$ increased, and it was also increased while

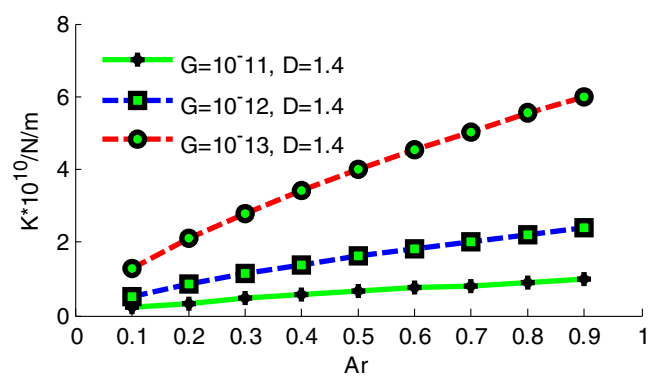

(a)

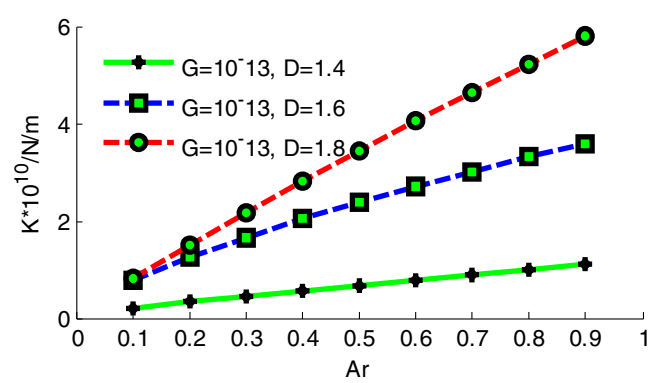

(b)

Fig. 8 Relationship of parameters $K_{\mathrm{s}}, A_{\mathrm{r}}, G$, and $D$ : a as $G$ (variable) and $D$ (constant); $\mathbf{b}$ as $G$ (constant) and $D$ (variable)

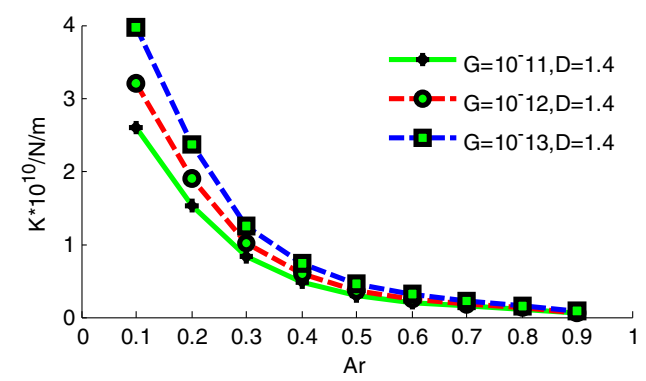

(a)

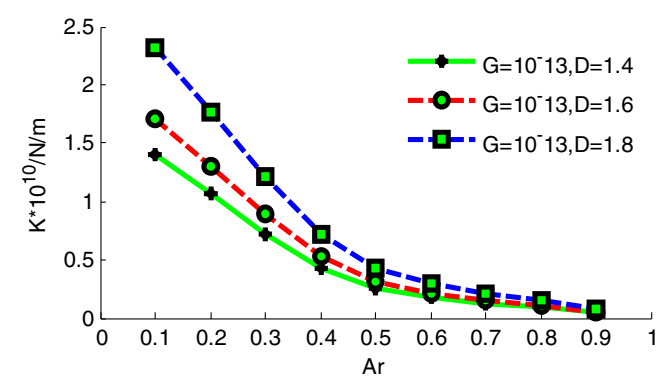

(b)

Fig. 9 Relationship of parameters $K_{\mathrm{L}}, A_{\mathrm{r}}, G$, and $D$ : a as $G$ (variable) and $D$ (constant); $\mathbf{b}$ as $G$ (constant) and $D$ (variable)

fractal dimension $D$ increased or fractal roughness coefficient $G$ decreased.

The variation of total equivalent stiffness of oily porous fixed joint interface is shown in Fig. 10. It is concluded from Fig. 10 that equivalent stiffness had a turning point at actual contact area $A_{\mathrm{r}}$ is about $30 \%$ of nominal contact area $A$. The

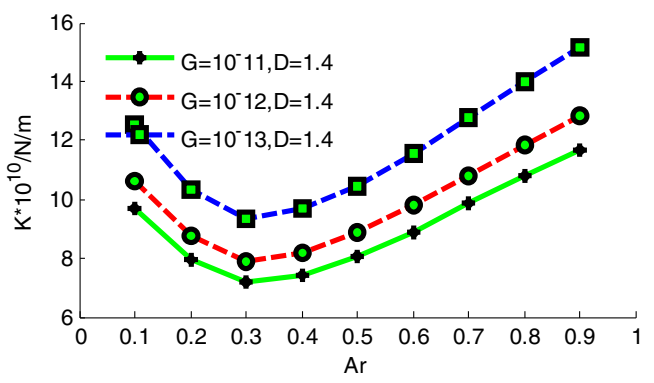

(a)

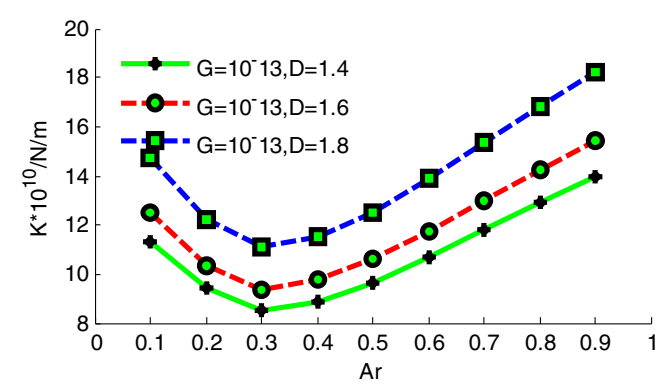

(b)

Fig. 10 Total equivalent stiffness along with $A_{\mathrm{r}}, G$, and $D$ : a as $G$ (variable) and $D$ (constant); b as $G$ (constant) and $D$ (variable) 


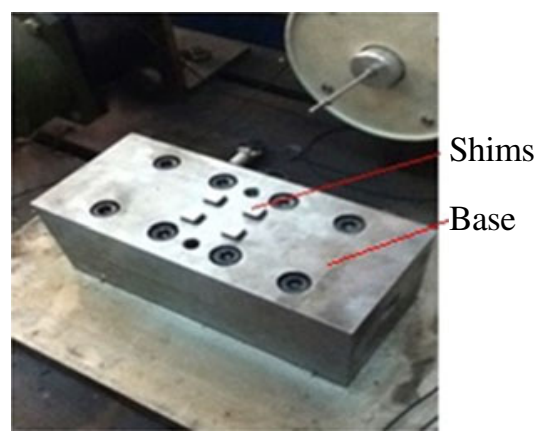

(a)

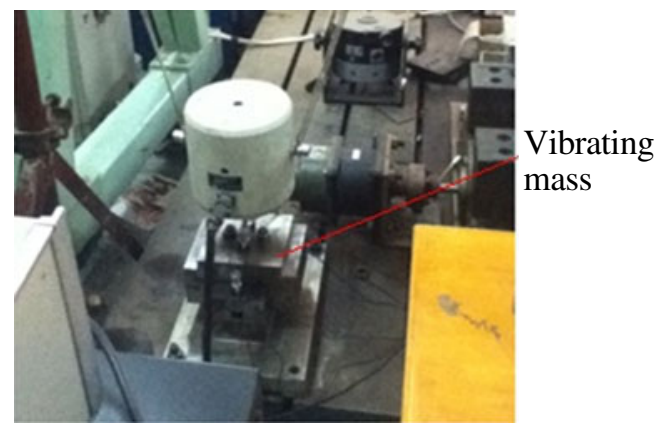

(b)

Fig. 11 Picture of designed experimental setup. a Before assembly. b After assembly

variation of total equivalent stiffness was also increased with $D$ increased and then decreased with $G$ increased.

The liquid stiffness plays a major role while real contact area is below $30 \%$ of nominal contact area, and the total equivalent stiffness of joint is mainly determined by oil film pressure. What is more, when real contact area is below $10 \%$ of nominal contact area, the liquid stiffness is equivalent to hydrostatic stiffness approximately. On the contrary, the solid stiffness plays a major role while real contact area is above $50 \%$ of nominal contact area, and the joint is equivalent to pure solid contact. However, the situation is difficult to achieve due to manufacturing constraints.

Table 3 Normal experimental results of two kinds of gaskets under different pre-tightening

\begin{tabular}{lllll}
\hline $\begin{array}{l}\text { Material } \\
\text { of joint }\end{array}$ & $\begin{array}{l}\text { Surface } \\
\text { roughness, } \\
\mu \mathrm{m}\end{array}$ & $\begin{array}{l}\text { Pre-tightening } \\
\text { torque of the } \\
\text { bolts }(\mathrm{Nm})\end{array}$ & $\begin{array}{l}\text { Normal } \\
\text { stiffness } \\
K_{\mathrm{n}}\left(\times 10^{12}\right) \\
\mathrm{N} / \mathrm{m}^{3}\end{array}$ & $\begin{array}{l}\text { Normal } \\
\text { damping } \\
C_{\mathrm{n}}\left(\times 10^{7}\right) \\
\mathrm{Ns} / \mathrm{m}^{3}\end{array}$ \\
\hline Steel-steel & 3.2 & 15 & 2.05 & 0.11 \\
& & 20 & 2.52 & 0.09 \\
& & 25 & 2.70 & 0.09 \\
$\begin{array}{c}\text { Steel-porous } \\
\text { material }\end{array}$ & 3.2 & 15 & 3.20 & 0.54 \\
& & 20 & 3.63 & 0.60 \\
\hline
\end{tabular}

From the discussion above, it has more practical engineering value to research the dynamic characteristics of oily joint while real contact area is in $30-50 \%$ of nominal contact area.

\section{Experiment}

Designed experimental setup is shown in Fig. 6. Experimental subjects were the joints constituted by the mass, the base, and the shims between them. There are two sets of experimental shims: 45 steel and Fe-based porous material, respectively. Roughness of the surface is $3.2 \mu \mathrm{m}$. The production place of the porous is Quebec, Canada, and its trademark is 4001 .

The density of the porous is $6.5 \times 10^{3} \mathrm{~kg} / \mathrm{m}^{3}$. The porosity is $15 \%$. The hardness is HRB50. The weight of the vibrating mass is $15 \mathrm{~kg}$. There were two bolts to be used for pretightening in normal direction, and the initial tightening torque was $10 \mathrm{Nm}$. The picture of specific experimental setup is shown in Fig. 11.

Experiment was carried out under different preload using the two sets of shims respectively. Before the experiment, the porous shims were soaked in $0.018 \mathrm{~Pa} \cdot \mathrm{s}$ lubricants for a week to ensure that the porous material is full of oil. The experimental result is shown in Table 3.

In Table 3, normal stiffness and damping are described in per unit area. From the experimental result, some conclusions are obtained as follows.

1. The joint which contains an oil film interlayer formed by porous material and steel is superior to the joint with non-media formed by steel-steel in stiffness and damping characteristics. In the case of the same preload, the former's stiffness was increased by about $30 \%$.

It is mainly because it would form the tiny oilresilient support body while oil film as an interlayer filled in the joint. The stiffness of this oil-resilient support body and solid asperity parallel superimposed to form the total equivalent stiffness of joint. Although the base body of Fe-based porous material has degradation of stiffness compared with the dense 45 steel, however, the oily porous joint has a better stiffness performance compared with non-media 45 steel joint due to the role of pressurized oil film in the limited slit.

2. In an oil-free joint, the damping coefficient is related to the number of plastic-deformed asperities. The change of normal load has a little effect on the number, thus the damping coefficient changes a little with normal load. However, the damping coefficient may be increased several times in the oily joint due to some energy that is dissipated in the tiny oil-resilient support body. From Table 1, we can see the damping is increased five to six times. 


\section{Conclusions}

This paper proposes a kind of joint interface which consists of oily porous material, and the advantages of this kind of joint are not only increasing the stiffness and damping, but to fill oil for a very long period is not needed. Theoretically, it derived the normal shunt modal of fixed oily joint interface using fractal and Hertz contact theory, which divides the total contact area into solid and liquid part. At first, stiffness and damping of solid are described by fractal parameters, and then, the liquid counterpart is deduced from the average flow of generalized Reynolds equation through the real contact area. It is concluded from simulation and calculation that the total equivalent stiffness of oily joint is increased with $D$ and decreased with an increased $G$.

An experiment was also carried out to compare the performance of the porous material-steel oily joint with the non-media steel-steel joint. It is verified that the joint which contains the oil film interlayer formed by porous and steel is superior to the joint with non-media formed by steel in stiffness and damping characteristics. In the case of the same preload, the former's stiffness was increased by about $30 \%$. Meanwhile, the damping was increased of five to six times.

The mechanism of the oily joint is also discussed. Because of the role of tiny oil-resilient support body, although the base body of Fe-based porous has degradation of stiffness compared with dense 45 steel, the oily porous joint has a better stiffness performance compared with non-media 45 steel joint due to the role of pressurized oil film in the limited slit.

Acknowledgments This research was sponsored by the National Key Projects of China under grant no. 2009ZX04014-036 and the Youth Natural Science Foundation of China under grant no. 51105006.

Open Access This article is distributed under the terms of the Creative Commons Attribution License which permits any use, distribution, and reproduction in any medium, provided the original author(s) and the source are credited.

\section{References}

1. Quinn DD (2012) Modal analysis of jointed structures. J Sound Vib 331(1):81-93
2. Miller JD, Quinn DD (2009) A two-sided interface model for dissipation in structural systems with frictional joints. J Sound Vib 321(1-2):201-219

3. Singh B, Nanda BK (2012) Identification of damping mechanism in layered and welded structures. Int J Mech Sci 63(1):37-47

4. Powałka B, Okulik T (2012) Dynamics of the guideway system founded on casting compound. Int J Adv Manuf Tech 59:1-7

5. Beards CF (1989) Damping in structural joints. Shock Vib Dig 21 (4):3-5

6. Yaxin S (2004) Modeling, identification and simulation of dynamics of structures with joints and interfaces. Dissertation, University of Illinois at Urbana-Champaign

7. Park SS, Chae J (2008) Joint identification of modular tools using a novel receptance coupling method. Int J Adv Manuf Tech 35 (11-12):1251-1262

8. Ozdemir N, Bilgin B (2009) Interfacial properties of diffusion bonded Ti-6Al-4V to AISI 304 stainless steel by inserting a $\mathrm{Cu}$ interlayer. Int J Adv Manuf Tech 41(5-6):519-526

9. Gouker RM, Gupta SK, Bruck HA, Holzschuh T (2006) Manufacturing of multi-material compliant mechanisms using multi-material molding. Int J Adv Manuf Tech 30(11-12):10491075

10. Sahin M (2009) Joining of stainless-steel and aluminum materials by friction welding. Int J Adv Manuf Tech 41(5-6):487-497

11. Wu LS (1999) Study on vibration behavior of sandwich structure with spring interlayer. Chin J Mech Eng 1:81-84, In Chinese

12. Thomposn AH, Katz AJ, Krohn CE (1987) The micro geometry and transport properties of sedimentary rock. Adv Phys 36(5): 625-694

13. Majumdar A, Bhushan B (1991) Fractal model of elastic-plastic contact between rough surfaces. ASME J Tribol 113(1):1-11

14. Qu CN (2012) Research on stiffness and damping characteristics of fixed oily porous materials joint interface. Adv Mater Res 422:575-579

15. Boming Y (2008) Analysis of flow in fractal porous media transactions of the ASME. Appl Mech Rev 61(5):1-19

16. Qu QW, Zhu J (1998) Analysis of flowing characteristic of fluid at the condition of thin film lubrication. Mech Sci Tech Aerosp Eng 17(2):203-205, In Chinese

17. Shuyun J, Yunjian ZH, Hua ZH (2010) A contact stiffness model of machined plane joint based on fractal theory. ASME J Tribol 132(1):1-7

18. You JM, Chen TN (2009) Fractal model for normal dynamic parameters of joint surfaces. J Xi'an Jiaotong Univ (China) 43 (9):91-94, In Chinese

19. Wen SH, Zhang XL, Wu MX, Wen XG, Wang PY (2009) Fractal model and simulation of normal contact stiffness of joint interfaces and its simulation [J]. Nongye Jixie Xuebao 40(11):197-202, In Chinese

20. Zhou GR, Ma J (1990) Fluid lubrication theory. Zhejiang University Press, HangZhou 